\title{
New perspectives in gaze sensitivity research
}

\author{
Gabrielle L. Davidson $^{1,2} \cdot$ Nicola S. Clayton ${ }^{1}$
}

Published online: 18 November 2015

(C) The Author(s) 2015. This article is published with open access at Springerlink.com

\begin{abstract}
Attending to where others are looking is thought to be of great adaptive benefit for animals when avoiding predators and interacting with group members. Many animals have been reported to respond to the gaze of others, by co-orienting their gaze with group members (gaze following) and/or responding fearfully to the gaze of predators or competitors (i.e., gaze aversion). Much of the literature has focused on the cognitive underpinnings of gaze sensitivity, namely whether animals have an understanding of the attention and visual perspectives in others. Yet there remain several unanswered questions regarding how animals learn to follow or avoid gaze and how experience may influence their behavioral responses. Many studies on the ontogeny of gaze sensitivity have shed light on how and when gaze abilities emerge and change across development, indicating the necessity to explore gaze sensitivity when animals are exposed to additional information from their environment as adults. Gaze aversion may be dependent upon experience and proximity to different predator types, other cues of predation risk, and the salience of gaze cues. Gaze following in the context of information transfer within social groups may also be dependent upon experience with group-members; therefore we propose novel means to explore the degree to which animals respond to gaze in a flexible manner, namely by inhibiting or enhancing gaze following responses. We hope this review will stimulate gaze sensitivity research to expand beyond the narrow scope of
\end{abstract}

Gabrielle L. Davidson

gd339@cam.ac.uk

1 Department of Psychology, University of Cambridge, Downing Street, Cambridge CB2 3EB, UK

2 School of Biological, Earth and Environmental Sciences, University College Cork, Distillery Fields Campus, Cork, Ireland investigating underlying cognitive mechanisms, and to explore how gaze cues may function to communicate information other than attention.

Keywords Gaze sensitivity - Attention attribution · Perspective taking · Gaze following · Gaze aversion . Communication

\section{Introduction}

Gaze sensitivity is the ability to respond to the presence, orientation, or movement of the head and eyes. Attending to gaze cues plays a critical role in human interactions, and being able to understand the referential nature of looking allows us to recognize where another individual's attention is directed, acknowledge that they may have different visual perspectives than our own, and infer that they may see and therefore know something about their world based on where they are looking. Humans, however, are not unique in their sensitivity to gaze. Gaze following (co-orienting gaze with a conspecific or human experimenter) has been reported in apes (e.g., Bräuer, Call, \& Tomasello, 2005), monkeys (e.g., Emery, Lorincz, Perrett, Oram, \& Baker, 1997), ungulates (e.g., Kaminski, Riedel, Call, \& Tomasello, 2005), dogs (e.g., Bräuer, Call, \& Tomasello, 2004), birds (e.g., ravens (Corvus corax), Bugnyar, Stowe, \& Heinrich, 2004), and reptiles (red-footed tortoise (Geochelone carbonaria), Wilkinson, Mandl, Bugnyar, \& Huber, 2010). Moreover, gaze aversion, in which animals respond fearfully to direct gaze, presumably because forward facing eyes are associated with predator attacks, has been reported in a similar breath of taxa including mammals (e.g., Coss, 1978b), birds (e.g., Carter, Lyons, Cole, \& Goldsmith, 2008; von Bayern \& Emery, 2009), reptiles (basking black iguana (Ctenosaura similis), Burger, 
Gochefeld, \& Murray Jr, 1991), and fish (African jewel fish (Hemichromis bimaculatus), Coss, 1979) (see Davidson, Butler, Fernández-Juricic, Thornton, \& Clayton, 2013, for review). Comparative psychologists have used gaze sensitivity as a paradigm to test whether animal responses to gaze are due to an understanding of the attention and perspective of others, or whether they are responding to physical features of gaze direction as salient cues (e.g., movement, shape, conspicuousness) (e.g., Povinelli \& Eddy, 1996; von Bayern \& Emery, 2009). Here we review recent findings in gaze sensitivity research that contribute to the ongoing debate regarding the presence or absence of cognitive abilities such as attention attribution and perspective-taking in animals. We conclude that gaze sensitivity paradigms are unlikely to resolve this issue because tasks are confounded by gaze cues that may facilitate associative learning mechanisms, or tap into species-specific perceptual biases. Nevertheless, we argue that studies on cognition, ontogeny, and socio-ecological factors in combination are important for understanding gaze sensitivity responses in animals. We highlight how studies of the ontogeny of gaze sensitivity demonstrate that experience and learning may shape behavioral responses to gaze. This has implications for understanding gaze sensitivity in animals in natural settings as social and predatory information will vary between species, and temporally within species. We highlight avenues of gaze research that are still in their infancy, namely how animals integrate predator gaze with other cues of risk, how gaze information is transferred between individuals in social groups, and how eye gaze may be used for signalling purposes that communicate information other than attention. We hope this review will stimulate a broader scope of research to advance our understanding of how cognition, perception, and experience shape gaze sensitivity responses in animals, and how this functions in naturalistic settings.

\section{Attention attribution and visual perspective taking}

One of the primary aims in gaze following and gaze aversion research is to establish whether animals comprehend what others can "see," namely whether they attribute attention or consider the visual perspective of others. In a gaze sensitivity context, attention attribution refers to the ability to infer where another individual's attention is directed based on where they are looking, and visual perspective taking refers to the ability to infer that others may see different things than one sees oneself (Flavell, 1974, 1978). These cognitive feats are distinct from responses associated with behavior-reading. For example, one individual may orient their gaze with a companion because they are coordinating their behavior to match that of others, independently of attention attribution or perspective taking (e.g., understanding that by seeing, others have mental representations of the external world). Two paradigms are prominent in the literature that have been adopted to distinguish between these alternative explanations. These tasks are the geometric gaze task and food choice tasks which aim to test whether subjects are aware that others can see things (e.g., food) that are hidden behind obstructions. In the geometric gaze task, an experimenter or a conspecific looks towards one side of a barrier, which is on the opposite side of the subject's position. In the "low-level" cognitive model, the subject should orient their gaze with that of the demonstrator and gaze towards the barrier only. In the "high-level" cognitive model, the subject should reposition themselves so they can see the other side of the barrier where the demonstrator is gazing (Povinelli \& Eddy, 1996). This would suggest that the subject recognizes that the barrier occludes their view, and that they must reposition themselves in order to see where the demonstrator's attention is fixed. In the food choice tasks, experiments are designed so that animals attending to an experimenter's direction of attention should use gaze direction to make their choice (i.e., between which experimenter to approach/avoid or where to search for hidden food).

Many of the gaze sensitivity tasks designed to test cognition in animals have reported varied results, demonstrating that some animals benefit from attending to certain gaze cues over others. Although many of these differences may be explained by socio-ecological differences (reviewed in Davidson et al., 2013), these functional explanations do not tell us whether animals are using gaze cues to read behavior or to infer attention. Northern bald ibises (Geronticus eremita) (Loretto, Schloegl, \& Bugnyar, 2010) and gibbons (Hylobates spp. and Symphalangus syndactylus) (Liebal \& Kaminski, 2012) fail the geometric gaze task. In contrast, all of the great apes (Bräuer et al., 2005), spider monkeys (Ateles geoffroyi) and capuchin monkeys (Cebus apella) (Amici, Aureli, Visalberghi, \& Call, 2009), dogs (Canus lupus) (Bräuer et al., 2004), and ravens (Bugnyar et al., 2004) gaze behind barriers, suggesting that they appreciate others' fields of view, and thus where their attention is directed. An alternative interpretation is that individuals may learn that repositioning themselves is associated with spotting something of interest on the other side of a visual obstruction. If the former interpretation were correct, we would expect animals to be capable of transferring their understanding of attentional states across a range of gaze tasks in different contexts. For many species, performance is not consistent. For example, when given the opportunity to direct begging gestures towards one of two human experimenters with different attentional states, chimpanzees (Pan troglodytes) did not differentiate between the two experimenters. Only after numerous trials did chimpanzees gradually learn to beg from the individual who could see them (Povinelli \& Giambrone, 2000). In a similar study, apes did not require learning to beg towards attentive humans, but they did so only if both body and face were directed towards them, and the subjects did not distinguish between open and closed 
eyes (Kaminski, Call, \& Tomasello 2004). In contrast, both dogs (Viranyi, Topal, Gacsi, Miklosi, \& Csanyi, 2004) and horses (Equus caballus) (Proops \& McComb, 2010) preferentially approached attentive humans in order to obtain food. Variation in performance within and between species is also apparent in the object choice task, where an experimenter indicates the location of hidden food by fixating their gaze on one of two cups. Jackdaws (Corvus monedula) (von Bayern \& Emery, 2009) and dogs (e.g., Hare \& Tomasello, 2005) have used gaze cues to correctly locate hidden food, whereas chimpanzees (Call, Hare, \& Tomasello, 1998), capuchins (Anderson, Sallaberry, \& Barbier, 1995), rhesus macaques (Macaca mulatta) (Anderson, Montant, \& Schmitt, 1996), domestic goats (Capra hircus) (Kaminski et al., 2005) ravens (Schloegl, Kotrschal, \& Bugnyar, 2008a), and horses (Proops, Walton, \& McComb, 2010) have been reported to perform at chance levels. Performance typically improves with pointing gestures or if the experimenter touches the correct location (capuchins, Anderson et al., 1995; chimpanzees, Call, Agnetta, \& Tomasello, 2000; dogs, Call, Brauer, Kaminski, \& Tomasello, 2003; domestic goats, Kaminski et al., 2005; ravens, Schloegl et al., 2008a; horses, Proops et al., 2010). It is difficult to conclude that animals failing the object choice task do not attribute attention to the experimenter based on gaze cues. This is because there are some criticisms regarding the validity of this task (e.g., Hare \& Tomasello, 2004; Mulcahy \& Hedge, 2012). For instance, animals' performance may be dependent on the subject's perception of the task. Primates and other animals may not understand the cooperative nature of the task, whereas domesticated animals such as dogs may be more attuned to humans indicating the location of hidden food (Hare, Brown, Williamson, \& Tomasello, 2002) (see also Mulcahy \& Hedge, 2012 on how design features of the object choice task influence results).

Nevertheless, some gaze sensitivity paradigms have been designed specifically to increase their ecologic validity. Tasks presented within a competitive framework or between conspecifics may better reflect their natural behavior. When given the opportunity to observe a competitor raven inspecting areas in an aviary where it had hidden food, ravens did not use this information to pilfer caches from these locations (Schloegl, Kotrschal, \& Bugnyar, 2008b). In contrast, free-ranging rhesus macaques did attend to gaze cues when stealing from humans as they preferred to take food located in front of experimenters who could not see them (gaze averted away, or eyes covered), than those that could (Flombaum \& Santos, 2005). The authors presented their results as evidence for macaques attributing mental states to the human experimenters (see also Bulloch, Boysen, \& Furlong 2008, for similar interpretations on visual attention in chimpanzees); however, macaques may have based their choice on the presence or absence of salient visual cues (i.e., two eyes) (Emery \& Clayton, 2008). These opposing interpretations highlight the hazards of applying gaze sensitivity paradigms as a means for testing attention attribution or perspective taking. All tests are inherently confounded by the presence of a cue; therefore making associative learning processes sufficient for explaining animal responses to gaze.

One way of controlling for responses to cues is to present subtly different gaze cues that are (in theory, see below) equally conspicuous, but represent different directions of attention. Human infants are capable of following subtle eye movements; whereas, chimpanzees are reliant on head direction, rather than eye direction (Tomasello, Hare, Lehmann, \& Call, 2007). These differences may be explained by speciesspecific perceptual differences, rather than cognitive differences. Humans may be more attentive to eye direction than other primates because the exposed white sclera around the iris and the horizontally elongated shape makes eye direction very easy to detect, and is thought to have evolved specifically for cooperative communication in humans (Kobayashi \& Kohshima 1997; Tomasello, Hare, Lehmann, \& Call 2007). Therefore it may be erroneous to conclude that chimpanzees are incapable of attention attribution. This is because they do not perceive eye direction as a meaningful cue to indicate gaze direction. Gaze aversion tasks have also tested whether birds can distinguish between subtle eye gaze cues (Hampton, 1994; Carter et al., 2008; Clucas, Marzluff, Mackovjak, \& Palmquist, 2013; Garland, Low, Armstrong, \& Burns, 2014; von Bayern \& Emery, 2009). Predators with gaze facing towards prey rather than away may predict a predator's attack; therefore prey should respond more aversively to eyes that are direct rather than averted. Jackdaws (von Bayern \& Emery, 2009), New Zealand robins (Petroica australis) (Garland et al., 2014), American crows (Corvus brachyrhynchos) (Clucas et al., 2013), and starlings (Sturnus vulgaris) (Carter et al., 2008), but not house sparrows (Passer domesticus) (Hampton, 1994), are sensitive to subtle differences in eye direction. Although in principle it may seem reasonable to assume that the capacity to differentiate between very subtle eye orientations would be an indication of where another individual is looking, it remains plausible that birds have evolved predispositions to respond fearfully to forwardfacing eyes, or individuals may have learned which cue was more likely to predict the actions of humans or natural predators. Birds may base the direction of the pupil relative to the boundaries of the eyes to predict a predator's direction of movement, independent from any concept of "looking" (albeit mistakes in the context of predation would be very costly). Indeed, there is evidence to suggest that conspicuous shapes in general invoke aversive behaviors in birds (e.g., Stevens, Hardman, \& Stubbings, 2008). A dark pupil and iris surrounded by white sclera may be perceived as highly conspicuous due to the clear boundary outline and highly contrasting features over $360^{\circ}$, compared to an eye directed to the side where the amount of boundary outline and contrasting 
colors would be comparatively less (Stevens, 2007; Stevens \& Ruxton, 2011) (Fig. 1). To date no studies have explicitly investigated whether eye shapes with a centered pupil/iris are perceived as more conspicuous than eye shapes with the pupil/iris positioned at the side. This would help to elucidate whether aversive responses to direct gaze are guided by visual processing mechanisms alone, rather than cognitive mechanisms. For example, if juvenile birds with no prior exposure to predator eyes respond most aversively to centered, rather than side-positioned pupil/iris, this would rule out associative learning or attention mechanisms.

\section{The ontogeny of gaze sensitivity}

As we have discussed, one of the fundamental difficulties in gaze sensitivity research is that tasks are confounded by the presence of a visual cue (i.e., gaze) that animals can respond to without the requirement of cognitive mechanisms such as attention attribution. Nevertheless, gaze research can be a useful tool for understanding inherited and learned responses and whether individuals are capable of responding flexibly depending on their experience and the context in which they are presented with gaze cues. Developmental studies of gaze following and gaze-aversion responses have provided insight into how gaze sensitivity emerges in juveniles, how gaze sensitivity is effected by early experience (i.e., exposure to gaze
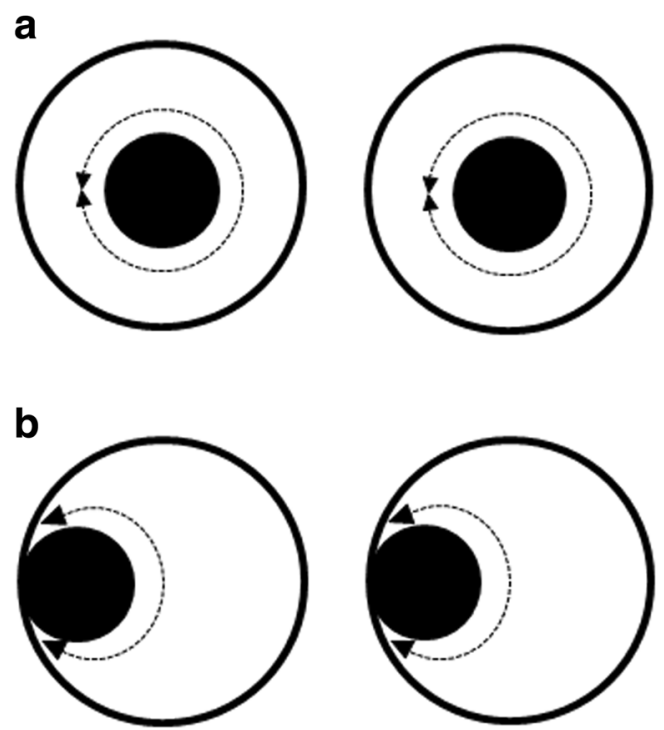

Fig. 1 Example of gaze stimuli representing eye-shapes with pupils (a) directed straight ahead and (b) to the side. Dotted arrows indicate the surface area where there is high contrast and a clear boundary outline between the pupil and the surrounding eye. This surface area is greater in the eyes straight than the eyes to the side stimulus. Therefore, eyes straight may be perceptually more conspicuous to prey species and thus elicit stronger aversive responses cues), and whether individuals habituate to gaze cues if they do not provide useful information.

Fearful responses to direct gaze cues emerge soon after hatching in chickens (Gallus gallus) (Jones, 1980) and in African jewel fish (Coss, 1979). Juvenile chicks discriminated between different features of eye-shapes (such as number of spots, pairedness, horizontal alignment), suggesting that discrimination of the most "eye-like" shapes is an innate response (Jones, 1980). This seemingly innate ability to recognize direct eye-shapes as fearful stimuli demonstrates that eye avoidance need not invoke any understanding of "seeing."

There is evidence indicating that early experience may influence the development of gaze-aversion responses. Bobwhite quail (Colinus virginianus) chicks that have had no prior experience with human faces did not avoid a human looking towards a food source; whereas individuals that had been exposed to human faces did avoid them (Jaime, Lopez, \& Lickliter, 2009). Further evidence to show that the development of gaze aversion may be influenced by experience comes from studies with jewel fish (Coss, Marks, \& Ramakrishnan, 2002). Those reared with conspecifics as opposed to eyeless cave fish displayed fearful responses to a model of an adult jewelfish with two horizontally placed black spots by 5 months of age, whereas the latter group did not respond until 7 months of age. Moreover, jewel fish decreased their aversive behavior to the same model as they became adults (Coss, 1978a). These development trajectories in jewel fish may function in juveniles to avoid predation when they are most at risk, and to engage in face-to-face combat with conspecific competitors as adults (Coss, 1978a, 1979). This study also demonstrates how aversion to eyes may function both in a predatory context, and during conflicts with conspecifics.

Ontogeny of gaze following has focused specifically on the emergence of gaze into distant space and geometric gaze. Rhesus macaques began to follow a human experimenter's gaze in mid-infancy (5.5 months) and chimpanzees by late infancy (3-4 years of age) (Tomasello, Hare, \& Fogleman, 2001). Juvenile pigtail-macaques (Macaca nemestrina) (2-6 years of age) also followed a human experimenter's head direction, and as adults ( $>6$ years of age) they began to follow eye movement independent of head movement (Ferrari, Kohler, Fogassi, \& Gallese, 2000). As great apes become older, they also engage more in "double check-backs," whereby they look back at the experimenter, presumably to re-assess where their gaze is directed (Brauer, Call \& Tomasello, 2005). Chimpanzees are capable of gazing behind barriers at 5 years of age (Povinelli \& Eddy, 1996), but our understanding of how geometric gaze develops in other animals is limited. Ravens have been reported to follow conspecific look-ups 6 days after fledging, co-orient with human gaze 8 weeks later, and gaze behind barriers around 6 months of life (Schloegl, Kotrschal, \& Bugnyar, 2007), and similar findings were 
reported for rooks (Corvus frugilegus) (Schloegl, Schmidt, Scheid, Kotrschal, \& Bugnyar, 2008). By comparison, Greylag geese (Anser anser) began following the gaze of conspecific look-ups earlier than ravens (10-43 days after hatching) (Kehmeier, Schloegl, Scheiber, \& Weiß, 2011). As precocial birds, greylag geese may benefit from looking up for predators soon after hatching compared to altricial ravens that would still be in their nest at this stage of life.

Overall, these studies show that following the gaze of another individual emerges very soon in life, suggesting that the co-orientation of gaze at this time is likely an example of fixed action patterns in response to stimuli (e.g., behavioral coordination), rather than cognitive mechanisms such as attention attribution and perspective taking. Gaze around barriers emerges later, but whether this coincides with the development of attention attribution (if at all), or whether it coincides with associative learning through experience (e.g., finding food or caching behind barriers) is an avenue of research that deserves further attention.

\section{Gaze sensitivity and learning}

The effect of early experience on the emergence of gaze following has yet to be explored; however, once gaze following has developed, studies have tested the effect of habituation to gaze cues ( Schloegl et al., 2007; Tomasello et al., 2001). Chimpanzees and ravens, but not macaques, will stop responding to a human repeatedly orienting their head and eye gaze towards a location with nothing of interest through habituation learning ( Schloegl et al., 2007; Tomasello et al., 2001). Therefore chimpanzees and ravens can use gaze following behavior in a flexible manner, by adjusting their response so that it matches the current social situation (Tomasello et al., 2001). This highlights the potential for further investigation into how responses to gaze cues may change throughout adulthood. This is important when making inferences about animal responses to gaze in terms of attention attribution, and for understanding the function of these behaviors in naturalistic settings when confronted with predators and when responding to conspecifics.

Defensive behavior in response to predators may change depending on the frequency, duration, or type of experience (e.g., Curio, Ernst, \& Vieth, 1978; Deecke, Slater, \& Ford, 2002; Wiebe, 2004; Marzluff, Walls, Cornell, Withey, \& Craig, 2010). This is particularly relevant in urban versus rural areas where human density differs (e.g., Moller, Grim, IbanezAlamoo, Marko, \& Tryjanowski, 2013). Therefore, habituation or close proximity with humans may greatly influence whether prey are ever close enough to a predator to perceive differences in gaze orientation or to associate different gaze cues with specific intentions or actions. For instance, animals may learn visual features of the eyes and that eyes forward predicts a human moving forward in the same direction, and that eyes to the side is generally not followed by a forwarddirected action. Birds that have been reported to discriminate between human eye directions have had extensive experience with humans. This is because they have either been raised by humans (jackdaws, von Bayern \& Emery, 2009), tested within in the confines of an experimental arena with the human (starlings, Carter et al., 2008; jackdaws, von Bayern \& Emery, 2009) or habituated to close observation (American crows, Clucas et al., 2013; New Zealand robins, Garland et al., 2014). Similarly, the type of predator may determine whether gaze direction is considered valued information (sensu Stephens, 1989). It may be more adaptive to flee at the mere sight of an especially threatening predator type than one that attacks less frequently. It is difficult to disentangle whether the presence (or lack) of responses to gaze cues are dependent upon habituation, association or distance constraints, but studies on wild animals and how they respond to the gaze of different predator types would help to clarify if discrimination between eye gaze direction is in fact an artefact of learning through close proximity to humans (albeit urban populations may be inherently bolder, as opposed to habituated to humans, e.g., Moller, 2008). Wild jackdaws discriminated between eyes open versus closed, but not eyes to the side when confronted with a model fox at a distance of 10 $\mathrm{m}$ (Davidson, 2014). Therefore in this context, eye direction may not be an informative cue for wild birds during an escape from predators.

\section{Gaze cues and additional predator and social information}

Additional variables that may influence whether animals attend to predator gaze cues may include indirect cues of predation risk. Indirect cues include landscape characteristics, such as distance to cover in the event of escape (Fong, Delong, Hogan, \& Blumstein, 2009), background coloration useful for camouflage (Thorson, Morgan, Brown, \& Normal, 1998), and density or numbers of individuals within a group (e.g., Fernandez-Juricic, Siller, \& Kacelnik, 2004). Some cues of predation risk may be more salient than others. For instance, in wild oldfield mice (Peromyscus polionotus), indirect cues (vegetative cover) influence feeding rates more than direct cues (predator urine) (Orrock, Danielson, \& Brinkerhoff, 2004). A recent study looked at whether American crows responded to human gaze direction and facial expression. Birds had a shorter flight initiation distance when an approaching human was looking directly at the crow, rather than away, regardless of the facial expression (scowling vs. smiling) (Clucas et al., 2013). Captive jackdaws showed adverse responses to direct gaze if the human was unfamiliar, but not when they were a familiar caretaker (von Bayern \& 
Emery, 2009). Furthermore, wild jackdaws integrated both predator identity (i.e., a human that had disturbed their nest from one that had not) and gaze direction (looking towards the nest rather than away), albeit human identity influenced their aversive behavior more than gaze direction (Davidson, Clayton, \& Thornton 2015). More studies regarding how animals integrate gaze cues with other indirect and direct cues of predation would provide a more complete picture of how and when animals use information from gaze cues to optimise decisions.

How animals value and act on information from gaze cues may be important in a gaze following context. Gaze following is an adaptive social behavior thought to provide information about predator location and hidden food location from conspecifics, yet how this behavior functions in a dynamic social group has received little attention. Context-dependent gaze following has been shown in long-tailed macaques (Macaca fascicularis), whereby responses were strongest when gaze cues were provided by socially meaningful facial expressions (i.e., bared teeth, rather than neutral expressions) (Goossens et al., 2008). This work could be expanded to ask whether individuals are capable of selectively reducing or increasing gaze following responses depending on who is providing the gaze cue. Being able to recognize individuals and remember past interactions is an important part of social living (e.g., Humphreys, 1976; Kondo, Izawa, \& Watanabe, 2012) and could be applied in a gaze following context. Consider individual A, a typically poor producer that rarely spots items of interest. If individuals can assign a category (e.g., poor producer/unreliable information) to members within their social group, we may expect habituation to individual A's gaze direction, while maintaining a response to the gaze of other companions that are better producers. In addition, whether an individual follows the gaze of another may be dependent upon how they obtain information. Animals may value their own personal information above social information (Dall, Giraldeau, Olsson, McNamara, \& Stephens, 2005). For example, if one group member gazed towards an area that an observer knew was recently depleted of food, the observer may inhibit their gaze following responses, having recently looked in the same location. From a cognitive perspective, being able to selectively respond to different cue-givers may not necessarily indicate attention attribution, but it would demonstrate a cognitive flexibility to incorporate information on when to attend to gaze cues, rather than co-orienting gaze in response to all gaze cues.

\section{Visual monitoring in groups}

The ability to follow the gaze of group members would be beneficial as a predator detection mechanism, which has the potential to function in a group vigilance context. Vigilance is typically quantified as the amount of time not foraging, time taken to forage a specified number of food items, immobility, crouching, and/or upright posture. The group size effect posits that individuals reduce vigilant behaviors as their group size increases because there are more individuals scanning for potential predators (Elgar, 1989; Lima, 1995). Group members may independently adjust their vigilance, for example, by following a rule associated with how many other individuals are in the group. Alternatively, under the collective detection hypothesis, individuals may monitor companions and adjust their vigilance based on what their neighbors are doing (e.g., Bahr \& Bekoff, 1999). Groups can either coordinate their vigilant behavior asynchronously (i.e., one or some individuals look up while others forage) or synchronously (i.e., vigilant behaviors are more likely to occur at the same time). Evidence for visual monitoring within foraging groups is mixed. Lima (1995) found no support that wild juncos (Junco hyemalis) and American tree sparrows (Spizella arborea) adjusted their vigilance when less vigilant members joined their group, and vigilance in a foraging pair of zebra finches (Taeniopygia guttata) did not differ if a partition did or did not block their view of their partner (Beauchamp, 2002). Other studies have used snapshots of group behavior to determine whether individuals were exhibiting vigilant behavior at the same time more or less than would be predicted statistically. These studies provide evidence that animals do monitor their neighbors, and tend of synchronize, rather than asynchronize their vigilance (Beauchamp, 2009; Ge, Beauchamp, \& Li, 2011; Fernandez-Juricic et al., 2004; Pays, Jarman, et al., 2007; Pays, Renaud, et al. 2007). We suggest that measuring gaze following could also provide a direct measure of visual monitoring. Times at which individual group members look up could be recorded sequentially and analyzed using social network analysis (e.g., Networkbased diffusion analysis, Franz \& Nunn, 2009) to determine whether information about vigilance is transferred socially, or whether individuals adjust their vigilant behaviors independently, for example in response to external stimuli (e.g., a predator or suspicious movement). Information about group size, distance between neighbors, species (in heterospecific groups), dominance status, kinship, etc. could be incorporated into these network models, many of which are factors known to influence vigilance (e.g., Beauchamp, 2003; FernandezJuricic \& Kacelnik, 2004; Fernandez-Juricic et al., 2004). In addition to testing the visual monitoring hypothesis, gaze following in a group foraging context could provide insight into how gaze following functions in naturalistic settings. If animals are reflexively responding to neighbor looks ups, we would predict gaze following to follow the synchronous model. If individuals are flexible in their gaze following responses, they may be capable of inhibiting gaze following behaviors, as would be predicted in the asynchronous model. These processes may also be dependent upon the variables listed above. 


\section{Alternative functions of eye communication}

So far we have discussed how gaze sensitivity may provide information about predation, location of hidden food or social interactions based on where others are orienting their gaze. In many of these cases it has been shown that the salience of the eyes may be an important cue. In humans, the conspicuous white sclera around the iris is a signal that helps to make the direction of attention visually salient (e.g., Kobayashi \& Kohshima, 1997; Tomasello, Hare, Lehmann, \& Call, 2007). However, there is evidence to suggest that conspicuous eyes may be important for communicating information other than gaze direction. For instance, unlike mammals that have smooth muscles controlling the pupil, birds have striated muscles allowing for voluntary control of pupil dilation (Bayón, 2007). Rapid pupil dilation and contraction independent of illuminance has been reported in birds (Gregory \& Hopkins, 1974; Mann, 1931). Moreover, species of guppies can rapidly change their eye color between silver to black (Martin \& Hengstebeck, 1981; Volpato, Luchiari, Duarte, Barreto, \& Ramanzini, 2003), yet the function of these visual changes in the eyes, the information they convey or how conspecifics respond is unknown. Many birds (e.g., Craig \& Hulley, 2004), amphibians (e.g., Amat, 2013), and fish have salient eyes, and the function of these colors as a means for communication represents an area worthy of future research. Several hypotheses have suggested that eye coloration may be related to ecology (Craig \& Hulley, 2004; Amat, 2013), aggression and dominance (e.g., Martin \& Hengstebeck, 1981; Craig, 1988; Volpato et al., 2003), mate recognition and/or sexual selection (e.g., Smith, 1967; Amat, 2013). In support of the sexual selection hypothesis, it has been proposed that the intensity of iris color in birds may be an honest indicator of metabolic efficiency through the anti-oxidant activities of hemoglobin or pterins present in red-colored irides (McGraw, 2006; Oliphant \& Hudon, 1993). To date, the most convincing evidence for a potential function of iris color in non-primates is that conspicuous eyes may be linked to nest guarding in jackdaws, to signal to competitors to keep away from occupied cavities (Davidson, Clayton, \& Thornton, 2014). This work represents limited progress, and we urge researchers to explore these potential functions for eye salience and communication.

\section{Conclusions}

It is clear that animals obtain important information from gaze cues, and the content of this information remains a primary focus in the literature. Specifically, studies aim to determine if animals are obtaining information regarding the attention and perspective of others. Not all animals are capable of gazing behind barriers, suggesting that perhaps this behavior requires some mental processes beyond associative learning. However, ecology and experience plays a role in how animals respond to gaze, providing individuals with the opportunity to learn to respond to gaze in the most adaptive way. Consequently, it remains difficult to assign underlying cognitive mechanisms with any degree of confidence, particularly if the physical properties of gaze cues provide salient cues to elicit appropriate responses independent of any concept of attention and visual perspective. Nevertheless, if learning plays a role in gaze sensitivity, then there is potential to investigate the degree of flexibility in gaze responses, and whether individuals have the capacity to change their responses as information from social group members, predation risk and other cues from the environment vary temporally. We hope that this review provides a foundation on which we can build our understanding of how animals perceive and process gaze cues, and how this information is encoded in conjunction with other informative cues related to predation risk and social interactions.

Acknowledgements We would like to thank two anonymous reviewers and the editor, Dr Crystal for their thoughtful and insightful feedback. GLD and NSC have received funding from the European Research Council under the European Union's Seventh Framework Programme (FP7/ 2007-2013) / ERC Grant Agreement No. 3399933.

Open Access This article is distributed under the terms of the Creative Commons Attribution 4.0 International License (http:// creativecommons.org/licenses/by/4.0/), which permits unrestricted use, distribution, and reproduction in any medium, provided you give appropriate credit to the original author(s) and the source, provide a link to the Creative Commons license, and indicate if changes were made.

\section{References}

Amat, F. W., Wollenberg, K. C., \& Vences, M. (2013). Correlates of eye colour and pattern in mantellid frogs. Salamandra, 49, 7-17.

Amici, F., Aureli, F., Visalberghi, E., \& Call, J. (2009). Spider monkeys (Ateles geoffroyi) and capuchin monkeys (Cebus apella) follow gaze around barriers: evidence for perspective taking? Journal of Comparative Psychology, 123, 368-374.

Anderson, J. R., Montant, M., \& Schmitt, D. (1996). Rhesus monkeys fail to use gaze direction as an experimenter-given cue in an objectchoice task. Behavioural Processes, 37, 47-55.

Anderson, J. R., Sallaberry, P., \& Barbier, H. (1995). Use of experimenter-given cues during object-choice tasks by capuchin monkeys. Animal Behaviour, 49, 201-208.

Bahr, D. B., Bekoff, M. (1999). Predicting flock vigilance from simple passerine interactions: modelling with cellular automata. Animal Behaviour, 58, 831-839.

Bayón, A., Almela, R. M., \& Talavera, J. (2007). Avian ophthalmology. Ophthalmology, 17, 1-13.

Beauchamp, G. (2002). Little evidence for visual monitoring of vigilance in zebra finches. Canadian Journal of Zoology, 80, 1634-1637.

Beauchamp, G. (2003). Group-size effects on vigilance: A search for mechanisms. Behavioural Processes, 63, 141-145.

Beauchamp, G. (2009). Sleeping gulls monitor the vigilance behaviour of their neighbours. Biology Letters, 5, 9-11. 
Bräuer, J., Call, J., \& Tomasello, M. (2004). Visual perspective taking in dogs (Canis familiaris) in the presence of barriers. Applied Animal Behaviour Science, 88, 299-317.

Bräuer, J., Call, J., \& Tomasello, M. (2005). All great ape species follow gaze to distant locations and around barriers. Journal of Comparative Psychology, 119, 145-154.

Bugnyar, T., Stowe, M., \& Heinrich, B. (2004). Ravens, Corvus corax, follow gaze direction of humans around obstacles. Proceedings of the Royal Society B, 271, 1331-1336.

Bulloch, M. J., Boysen, S., \& Furlong, E. E. (2008). Visual attention and its relation to knowledge states in chimpanzees, Pan troglodytes. Animal Behaviour, 76, 1147-1155.

Burger, J., Gochefeld, M., \& Murray, B. G., Jr. (1991). Role of a predator's eye size in risk perception by basking black iguana, Ctenosaura similis. Animal Behaviour, 42, 471-476.

Call, J., Agnetta, B., \& Tomasello, M. (2000). Cues that chimpanzees do and do not use to find hidden objects. Animal Cognition, 3, 23-34.

Call, J., Brauer, J., Kaminski, J., \& Tomasello, M. (2003). Domestic dogs (Canis familiaris) are sensitive to the attentional state of humans. Journal of Comparative Psychology, 117, 257-263.

Call, J., Hare, B. A., \& Tomasello, M. (1998). Chimpanzee gaze following in an object-choice task. Animal Cognition, 1, 89-99.

Carter, J., Lyons, N. J., Cole, H. L., \& Goldsmith, A. R. (2008). Subtle cues of predation risk: Starlings respond to a predator's direction of eye-gaze. Proceedings of the Royal Society B, 275, 1709-1715.

Clucas, B., Marzluff, J. M., Mackovjak, D., \& Palmquist, I. (2013). Do American crows pay attention to human gaze and facial expressions? Ethology, 119, 296-302.

Coss, R. G. (1978a). Development of face aversion by the jewel fish (Hemichromis bimaculatus). Zeitschrift für Tierpsychologie, 48, 28-46.

Coss, R. G. (1978b). Perceptual determinants of gaze aversion by the lesser mouse lemus (Microcebus murinus), the role of two facing eyes. Behaviour, 64, 248-270.

Coss, R. G. (1979). Delayed plasticity of an instinct: Recognition and avoidance of 2 facing eyes by the jewel fish. Developmental Psychobiology, 12, 335-345.

Coss, R. G., Marks, S., \& Ramakrishnan, U. (2002). Early environment shapes the development of gaze aversion by wild bonnet macaques (Macaca radiata). Primates, 43, 217-222.

Craig, A. J. F. K. (1988). Allofeeding and dominance interactions in the cooperatively breeding pied starling. Animal Behaviour, 36, 12511253.

Craig, A. J. F. K., \& Hulley, P. E. (2004). Iris colour in passerine birds: Why be bright-eyed? South African Journal of Science, 100, 584 588 .

Curio, E., Ernst, U., \& Vieth, W. (1978). Cultural transmission of enemy recognition: One function of mobbing. Science, 202, 899-901.

Dall, S. R. X., Giraldeau, L. A., Olsson, O., McNamara, J. M., \& Stephens, D. W. (2005). Information and its use by animals in evolutionary ecology. Trends in Ecology \& Evolution, 20, 187-193.

Davidson, G. L. (2014). Functions of gaze sensitivity and iris colour in birds. UK: University of Cambridge.

Davidson, G. L., Butler, S., Fernández-Juricic, E., Thornton, A., \& Clayton, N. S. (2013). Gaze sensitivity: Function and mechanisms from sensory and cognitive perspectives. Animal Behaviour, 87, 315.

Davidson, G. L., Clayton, N. S., \& Thornton, A. (2014). Salient eyes deter conspecific nest intruders in wild jackdaws (Corvus monedula). Biology Letters, 10.

Davidson, G. L., Clayton, N. S., \& Thornton, A. (2015). Wild jackdaws (Corvus monedula) recognise individual humans and may respond to gaze direction with defensive behaviour. Animal Behaviour, 108, $17-24$.
Deecke, V. B., Slater, P. J., \& Ford, J. K. (2002). Selective habituation shapes acoustic predator recognition in harbour seals. Nature, 420 , $171-173$.

Elgar, M. A. (1989). Predator vigilance and group size in mammals and birds: A critical review of the empirical evidence. Biological Reviews, 64, 13-33.

Emery, N. J., \& Clayton, N. S. (2008). Comparative social cognition. Annual Review of Psychology, 60, 87-113.

Emery, N. J., Lorincz, E. N., Perrett, D. I., Oram, M. W., \& Baker, C. I. (1997). Gaze following and joint attention in rhesus monkeys (Macaca mulatta). Journal of Comparative Psychology, 111, 286293.

Fernandez-Juricic, E., \& Kacelnik, A. (2004). Information transfer and gain in flocks: The effects of quantity and quality of social information at different neighbour distances. Behavioral Ecology and Sociobiology, 55, 502-511.

Fernandez-Juricic, E., Siller, S., \& Kacelnik, A. (2004). Flock density, social foraging, and scanning: An experiment with starlings. Behavioral Ecology, 15, 371-379.

Ferrari, P. F., Kohler, E., Fogassi, L., \& Gallese, V. (2000). The ability to follow eye gaze and its emergence during development in macaque monkeys. Proceedings of the National Academy of Sciences, 97, 13997-14002.

Flavell, J. (1974). The development of inferences about others. In T. Mischel (Ed.), Understanding Other Persons (pp. 66-116). Oxford: Blackwell.

Flavell, J. (1978). The development of knowledge about visual perception. In C. B. Keasey (Ed.), The Nebraska Symposion on Motivation (Vol. 25., pp. 43-76). Lincoln, NE: University of Nebraska Press.

Flombaum, J. I., \& Santos, L. R. (2005). Rhesus monkeys attribute perceptions to others. Current Biology, 15, 447-452.

Fong, T. E., Delong, T. W., Hogan, S. B., \& Blumstein, D. T. (2009). The importance of indirect cues for white-browed sparrow-weaver (Plocepasser mahali) risk assessment. Acta Ethologica, 12, 79-85.

Franz, M., \& Nunn, C. L. (2009). Network-based diffusion analysis: A new method for detecting social learning. Proceedings of the Royal Society B, 267, 1829-1836.

Garland, A., Low, J., Armstrong, N., \& Burns, K. (2014). Wild robins (Petroica longipes) respond to human gaze. Animal Cognition, 1-8.

Ge, C., Beauchamp, G., \& Li, Z. (2011). Coordination and synchronisation of anti-predation vigilance in two crane species. PLoS One, 6, e26447.

Goossens, B. M. A., Dekleva, M., Reader, S. M., Sterk, E. H. M., \& Bolhuis, J. J. (2008). Gaze following in monkeys is modulated by observed facial expressions. Animal Behaviour, 75, 1673-1681.

Gregory, R., \& Hopkins, P. (1974). Pupils of a talking parrot. Nature, 252, 637-638.

Hampton, R. R. (1994). Sensitivity to information specifying the line of gaze of humans in sparrows (Passer domesticus). Behaviour, 130, $41-51$.

Hare, B., Brown, M., Williamson, C., \& Tomasello, M. (2002). The domestication of social cognition in dogs. Science, 298, 1634-1636.

Hare, B. \& Tomasello, M. (2004). Chimpanzees are more skilful in competitive than in cooperative cognitive tasks. Animal Behaviour, 68, 571-581.

Hare, B., \& Tomasello, M. (2005). Human-like social skills in dogs? Trends in Cognitive Sciences, 9, 439-444.

Humphreys, N. K. (1976). The social functions of intellect. In P. P. Bateson \& R. A. Hinde (Eds.), Growing Points in Ethology (pp. 303-317). Cambridge: Cambridge University Press.

Jaime, M., Lopez, J. P., \& Lickliter, R. (2009). Bobwhite quail (Colinus virginianus) hatchlings track the direction of human gaze. Animal Cognition, 12, 559-565.

Jones, R. B. (1980). Reactions of male domestic chicks to twodimensional eye-like shapes. Animal Behaviour, 28, 212-218. 
Kaminski, J., Call, J., \& Tomasello, M. (2004). Body orientation and face orientation: Two factors controlling apes' begging behavior from humans. Animal Cognition, 7, 216-223.

Kaminski, J., Riedel, J., Call, J., \& Tomasello, M. (2005). Domestic goats, Capra hircus, follow gaze direction and use social cues in an object choice task. Animal Behaviour, 69, 11-18.

Kehmeier, S., Schloegl, C., Scheiber, I., \& Weiß, B. M. (2011). Early development of gaze following into distant space in juvenile Greylag geese (Anser anser). Animal Cognition, 14, 477-485.

Kobayashi, H., \& Kohshima, S. (1997). Unique morphology of the human eye. Nature, 387, 767-768.

Kondo, N., Izawa, E. I., \& Watanabe, S. (2012). Crows cross-modally recognize group members but not non-group members. Proceedings of the Royal Society B, 278, 1937-1942.

Liebal, K., \& Kaminski, J. (2012). Gibbons (Hylobates pileatus, H. moloch, H. lar, Symphalangus syndactylus) follow human gaze, but do not take the visual perspective of others. Animal Cognition, 15, 1211-1216.

Lima, S. L. (1995). Back to the basics of anti-predatory vigilance: The group-size effect. Animal Behaviour, 49, 11-20.

Loretto, M. C., Schloegl, C., \& Bugnyar, T. (2010). Northern bald ibises follow others' gaze into distant space but not behind barriers. Biology Letters, 6, 14-17.

Mann, I. (1931). Iris pattern in the vertebrates. The Transactions of the Zoological Society of London, 21, 355-412.

Martin, F. D., \& Hengstebeck, M. F. (1981). Eye colour and aggression in juvenile guppies, Poecilia reticulata peters (Pisces: Poeciliidae). Animal Behaviour, 29, 325-331.

Marzluff, J. M., Walls, J., Cornell, H. N., Withey, J. C., \& Craig, D. P. (2010). Lasting recognition of threatening people by wild American crows. Animal Behaviour, 79, 699-707.

McGraw, K. J. (2006). Mechanics of uncommon colors: Pterins, porphyrins, and psittacofulvins. In G. E. Hill \& K. J. McGraw (Eds.), Bird coloration. Volume I: Mechanisms and Measurements (pp. 358398). Cambridge, Massachusetts: Harvard University Press.

Moller, A. P. (2008). Flight distance of urban birds, predation, and selection for urban life. Behavioral Ecology and Sociobiology, 63, 63-75.

Moller, A. P., Grim, T., Ibanez-Alamoo, J. D., Marko, G., \& Tryjanowski, P. (2013). Change in flight initiation distance between urban and rural habitats following a cold winter. Behavioral Ecology.

Mulcahy, N. J., \& Hedge, V. (2012). Are great apes tested with an abject object-choice task? Animal Behaviour, 83, 313-321.

Oliphant, L. W., \& Hudon, J. (1993). Pteridines as reflecting pigments and components of reflecting organelles in vertebrates. Pigment Cell Research, 6, 205-208.

Orrock, J. L., Danielson, B. J., \& Brinkerhoff, R. J. (2004). Rodent foraging is affected by indirect, but not by direct, cues of predation risk. Behavioral Ecology, 15, 433-437.

Pays, O., Jarman, P. J., Loisel, P., \& Gerard, J. F. (2007). Coordination, independence or synchronization of individual vigilance in the eastern grey kangaroo? Animal Behaviour, 73, 595-604.

Pays, O., Renaud, P. C., Loisel, P., Petit, M., Gerard, J. F., \& Jarman, P. J. (2007). Prey synchronize their vigilant behaviour with other group members. Proceedings of the Royal Society B: Biological Sciences, 274, 1287-1291.

Povinelli, D. J., \& Eddy, T. J. (1996). Chimpanzees: Joint visual attention. Psychological Science, 7, 129-135.

Povinelli, D. J., \& Giambrone, S. (2000). Escaping the argument by analogy. In D. J. Povinelli (Ed.), Folk Physics for Apes (pp. 9-72). Oxford: Oxford University Press.
Proops, L., \& McComb, K. (2010). Attributing attention: the use of human-given cues by domestic horses (Equus caballus). Animal Cognition, 13, 197-205.

Proops, L., Walton, M., \& McComb, K. (2010). The use of human-given cues by domestic horses, Equus caballus, during an object choice task. Animal Behaviour, 79, 1205-1209.

Schloegl, C., Kotrschal, K., \& Bugnyar, T. (2007). Gaze following in common ravens, Corvus corax: Ontogeny and habituation. Animal Behaviour, 74, 769-778.

Schloegl, C., Kotrschal, K., \& Bugnyar, T. (2008a). Do common ravens (Corvus corax) rely on human or conspecific gaze cues to detect hidden food? Animal Cognition, 11, 231-241.

Schloegl, C., Kotrschal, K., \& Bugnyar, T. (2008b). Modifying the object-choice task: Is the way you look important for ravens? Behavioural Processes, 77, 61-65.

Schloegl, C., Schmidt, J., Scheid, C., Kotrschal, K., \& Bugnyar, T. (2008c). Gaze following in non-human animals: The corvid example. In E. A. Weber \& L. H. Krause (Eds.), Animal Behaviour: New Research (pp. 73-92). New York: Nova Science Publishers.

Smith, N. G. (1967). Visual isolation in gulls. Scientific American, 217, 94-102.

Stephens, D. W. (1989). Variance and the value of information. The American Naturalist, 134, 128-140.

Stevens, M. (2007). Predator perception and the interrelation between different forms of protective coloration. Proceedings of the Royal Society B: Biological Sciences, 274, 1457-1464.

Stevens, M., Hardman, C. J., \& Stubbings, C. L. (2008). Conspicuousness, not eye mimicry, makes "eyespots" effective antipredator signals. Behavioural Ecology, 19, 525-531.

Stevens, M., \& Ruxton, G. D. (2011). Linking the evolution and form of warning coloration in nature. Proceedings of the Royal Society of London B: Biological Sciences, 279, 417-426.

Thorson, J. M., Morgan, R. A., Brown, J. S., \& Normal, J. E. (1998). Direct and indirect cues of predatory risk and patch use by fox squirrels and thirteen-lined ground squirrels. Behavioural Ecology, 9, 151-157.

Tomasello, M., Hare, B., Lehmann, H., \& Call, J. (2007). Reliance on head versus eyes in the gaze following of great apes and human infants: The cooperative eye hypothesis. Journal of Human Evolution, 52, 314-320

Tomasello, M., Hare, B., \& Fogleman, T. (2001). The ontogeny of gaze following in chimpanzees, Pan troglodytes, and rhesus macaques, Macaca mulatta. Animal Behaviour, 61, 335-343.

Viranyi, Z., Topal, J., Gacsi, M., Miklosi, A., \& Csanyi, V. (2004). Dogs respond appropriately to cues of humans $\ni$ attentional focus. Behavioural Processes, 66, 161-172.

Volpato, G. L., Luchiari, A. C., Duarte, C. R. A., Barreto, R. E., \& Ramanzini, G. C. (2003). Eye color as an indicator of social rank in the fish Nile tilapia. Brazilian Journal of Medical and Biological Research, 36, 1659-1663.

von Bayern, A. M., \& Emery, N. J. (2009). Jackdaws respond to human attentional states and communicative cues in different contexts. Current Biology, 19, 602-606.

Wiebe, K. L. (2004). Innate and learned components of defence by flickers against a novel nest competitor, the European starling. Ethology, 110, 779-791.

Wilkinson, A., Mandl, I., Bugnyar, T., \& Huber, L. (2010). Gaze following in the red-footed tortoise (Geochelone carbonaria). Animal Cognition, 13, 765-769. 